\title{
FLOW BALANCED ROUTING IN WIRELESS SENSOR NETWORKS
}

\author{
Ashok Kumar.S ${ }^{1}$, Vanitha. $V^{2}$ \\ ${ }^{1}$ P.G. Scholar, Computer Science and Engineering, Kumaraguru College of Technology, Tamilnadu, India \\ ${ }^{2}$ Senior Associate Professor, Computer Science and Engineering, Kumaraguru College of Technology, Tamilnadu, India
}

\begin{abstract}
Wireless Sensor Networks (WSNs) consists of a large number of sensor nodes which are densely deployed. The clusters are formed based on the overlapping degree and residual energy of sensor and then hierarchical backbone is constructed with sink at the top. Flow Balanced Routing (FBR) overcomes the problem of imbalance in the energy consumption of Cluster Head(CH) nodes by sending the data in multiple paths, thereby increasing the network lifetime. If the data is sent in a single path to the sink, then the energy of the $\mathrm{CH}$ nodes will exhaust in that path. To avoid this, the data is sent in multiple path, by which the energy consumption of all the CH node are balanced. This approach enhances the lifespan of $\mathrm{CH}$ nodes, thereby increasing the network lifetime but it increases the delay and decrease the throughput. Since the CH's at higher level have large amount of data than CH's at lower level, the wake-up time of CH's at higher level is set to be higher than the CH's at lower level, which decrease the delay and also increases the network lifetime. Simulation results show that the proposed algorithm drastically increases the network lifetime and also decrease the end-toend delay.
\end{abstract}

Keywords: Clustering, Delay, Multiple Path, Network lifetime, Wake-up time

\section{INTRODUCTION}

A Wireless Sensor Network (WSN) consists of a large number of sensor nodes which are densely deployed. Sensors are used for sensing the environment. Since the coverage area of each sensor is very less, a network of sensors is needed to monitor the large environment. The WSN architecture is shown in Fig1

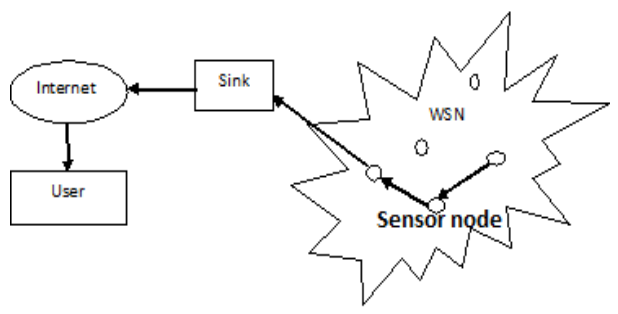

Fig-1: WSN architecture

The WSNs is widely used in military applications such as battlefield surveillance. It is also used in environment surveillance, defense monitoring, trading and customer applications.

\subsection{Routing}

Routing is the process of selecting best paths in a network[1]. Routing is performed for many kinds of networks, such as telephone network, electronic data networks and transportation networks. Most routing algorithms use only one network path at a time. . If multiple alternative paths is used for transmitting the data, energy of each sensor node get balanced. There are several routing techniques in Wireless sensor networks. In Flat routing protocol, each node typically plays the same role and sensor nodes collaborate together to perform the sensing task. In a hierarchical architecture, higher energy nodes are used to process and send the information and lower energy nodes are used in performing sensing of the target. Hierarchical routing is a efficient way to reduce the energy consumption by performing data aggregation and fusion which helps in reducing the number of transmitted messages to the BS. In Location based routing, the distance between neighboring nodes is estimated based on incoming signal strengths. In order to save energy, in some location based schemes nodes are in sleep mode, if there is no task.

\section{RELATED WORK}

This section deals with techniques like cluster head election, backbone construction, multi hop data transmission, and describe how the proposed protocol differs from them. Most of the cluster head selection approaches [2], [5] are designed with the goal to provide balanced energy consumption among sensor nodes, but at the same time, these approaches does not consider the network's requirement for full coverage over extended periods of time. The coverage aware cost metric considers the node's remaining energy as well as the coverage redundancy of its sensing range, thereby measuring the contribution of this node to the network coverage task. The sensors that are more important to the network coverage task are less likely to be selected as $\mathrm{CH}$ nodes. The HEED clustering protocol[7] uses a hybrid criterion for $\mathrm{CH}$ selection, which considers the residual energy of the node and the node's 
proximity to its neighbors or the node's neighbor degree. HEED prolongs the network lifetime by ensuring balanced energy dissipation as well as uniform distribution of cluster head nodes in network that contains uniformly dispersed sensor nodes. Hence the energy dissipation among the nodes are balanced which extends the network lifetime. The backbone members are selected based on the remaining energy of nodes[8]. The nodes with high remaining energy will be selected to relay the message to the sink. These nodes are the backbone nodes or CH's. Each node checks if it is a backbone member only based on the local information of the node. The MCDS[9] is built to reduce the number of nodes and links involved in communication. If the workload on each dominator in a CDS is not balanced, the dominators with high load (i.e.,) the dominator with large number of dominatees, deplete their energy quickly. Due to this the whole network might be disconnected. Hence, in addition of constructing a MCDS, it is also necessary to construct a Load-Balanced CDS (LBCDS)[10]. LEACH protocol is a cluster based routing protocol. In this, the CH's are selected randomly for even energy consumption among all the nodes, so that the overall lifetime of the network is prolonged[12]. However, the randomness in the selection of the $\mathrm{CH}$ 's may result in some poor clustering schemes. The clusters are formed only in the direction of sink and the shortest path from the area where the event occurs to the sink. A sensor has a higher chance of being a $\mathrm{CH}$, if it is closer to the sink, because Event-to-sink Directed Clustering [15] is designed to reach the sink using the shortest path to reduce energy consumption, delay and overhead. It selects $\mathrm{CHs}$ to be as close to the centre of the region where the event occurs as possible in order to make use of spatial correlation of data and efficient data aggregation and provides best coverage. The energy-efficient protocols[16],[17] are designed for WSNs, where sensors consume distinct energy in distinct states such as transmitting, receiving, sleeping, listening, idle and it also consume energy for state transition. TDMA is used as the MAC layer protocol and scheduling of sensor nodes is done with consecutive time slots at different radio states. Hence the number of state transitions is reduced. When the data is to be sent, any sensor node will wake up at most twice. Once for receiving all the packets from its children nodes and another for sending its own data to its parent node. Since the proposed algorithm considers the overlapping degree and residual energy in choosing the CH's, it minimize the death of the sensor. The Backbone constructed is not a simple tree. Each node may have multiple parents and hence the data is transmitted in multiple paths, which helps in balancing the energy of the CH's. The delay aware wake up scheduling scheme [18] is used to decrease the end-to-end delay which also increases the network lifetime.

\section{PROPOSED WORK}

The clusters are formed based on the overlapping degree and residual energy of sensor and then hierarchical backbone is constructed with sink at the top. The proposed system architecture is shown in Fig-2. Each $\mathrm{CH}$ node collects the data from its member nodes and performs aggregation and sends the collected data to sink by the multiple paths thereby the energy of the $\mathrm{CH}$ nodes is balanced. Each node determines the capable parents to send the data and send the data to their parents. Similarly, the data is sent to the parents at different levels.

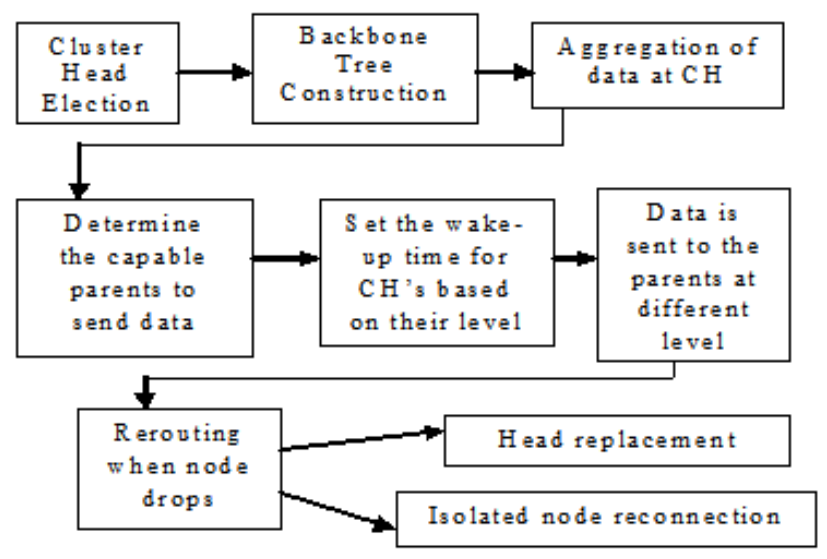

Fig-2: Proposed System Architecture

Since the CH's at higher level have large amount of data than $\mathrm{CH}$ 's at lower level, the wake-up time of CH's at higher level is set to be higher than the $\mathrm{CH}$ 's at lower level, which decrease the delay and also increases the network lifetime. The backbone is reconfigured only when the topology changes occur. It consists of 2 phases: Head replacement and Isolated node reconnection.

\subsection{Proposed Algorithms}

\subsubsection{Cluster Formation Algorithm}

Initially there are no clusters and all the sensor nodes are randomly deployed. Each node checks independently whether it can form cluster based on the percentage of remaining energy and its overlapping degree. If the overlapping degree of itself is greater than all its neighbors and if it is a node with higher energy than all its neighbors, it can send the $\mathrm{CH}$ announcement message to all its neighbors, only if it does not belong to any other cluster and if it is not in the transmission range of any other neighbor. When the $\mathrm{CH}$ announcement message is received by any node, it becomes a cluster member of that $\mathrm{CH}$ node if it does not belong to any other cluster and if it is not a waiting node. In most of the existing approaches, cluster formation is done periodically. Hence the CH's may change at each round. In general, the $\mathrm{CH}$ nodes at higher level need to send more data to the sink node. So the higher level $\mathrm{CH}$ node will be closer to the sink in order to optimize the energy consumption. If the $\mathrm{CHs}$ are elected periodically and $\mathrm{CHs}$ are distributed throughout the network, then the higher level $\mathrm{CH}$ may be changed to some other location which is far 
from the sink. Since the position of the node gets changed which is farther to the sink, it needs more energy to transmit all the data to the sink node. Since the energy of the sensor nodes are limited, the node's energy may get exhausted, which may lead to the death of the sensor node. In the existing protocols, even if the data transmission is not there, the Cluster formation is done periodically. But in the proposed algorithm, the Cluster formation is done only once at the initial stage. The cluster formation is done only when the CH's energy became less than the threshold value. Hence the network lifetime is increased.

\subsubsection{Backbone Construction Algorithm}

The clusters are formed by using cluster formation algorithm. The sink initially checks whether the number of clusters is equal to the last cluster count. If the energy of the $\mathrm{CH}$ exhausts or became lower than the predefined threshold, then the cluster formation is done again and the number of clusters may change. Hence the sink updates the last cluster count as the current cluster count. Hence the sink knows the number of clusters in the network. The sink sends the backbone construction message to all its neighbors which is one hop distance from it, with its level and its id respectively. Each neighbor node which receives the message, send the ON message to the sink which inform that it exist in the backbone. The neighbor node which receives the message, broadcast the backbone construction message to the neighbor. In the similar way all the nodes are added to the backbone network. The backbone construction is shown in Fig-3. When a particular backbone node $(\mathrm{CH})$ receives the Backbone construction message from more than one backbone node, it accept the node as parent which is at the lowest level. One backbone node can have multiple paths and once the node is added to the backbone its parents are not changed. If some of the $\mathrm{CH}$ nodes are not added to the network by one hop transmission distance, the sink increment the number of hops as two and it retransmit the message to neighbors. If the neighbor has already received the message, it simply broadcast to the neighbors with number of hops set as two. The sink will increment the number of hops until all the backbone nodes are added to the network.

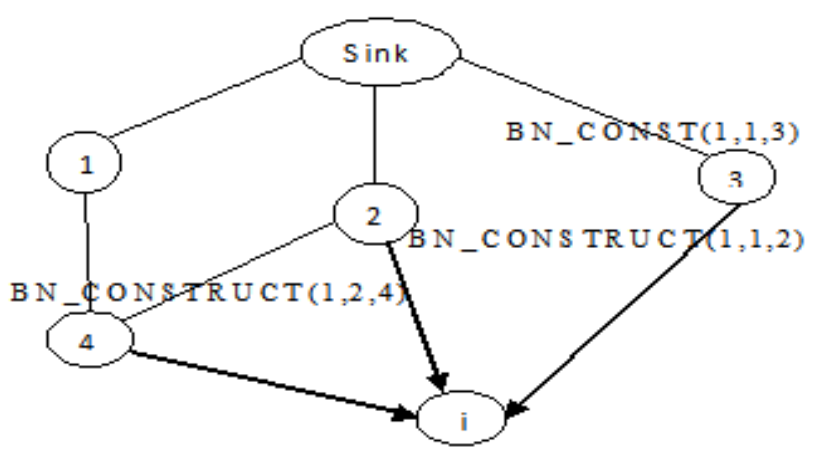

Fig-3: Backbone Construction
Since each backbone node may have multiple parents, the data is sent in multiple paths to the sink, which balances the energy consumption of the $\mathrm{CH}$ nodes which extends the lifetime of the $\mathrm{CH}$ node thereby the network lifetime is increased.

\subsubsection{Flow Balanced Routing Algorithm}

The backbone network is constructed with sink at the top. Each $\mathrm{CH}$ node collects the data from its member nodes and performs aggregation and sends the collected data to sink by the multiple paths. Since the data is sent in multiple paths to the sink, the energy is balanced among all the $\mathrm{CH}$ nodes which increase the network lifetime. Consider a Backbone node $(\mathrm{CH})$ i, which need to send I-bit of data to the sink, by multiple paths through its parents which is shown in Fig-4. Let the current energy of its three parents be $0.1 \mathrm{~J}, 0.3 \mathrm{~J}$ and $0.4 \mathrm{~J}$ respectively. After the data is transferred, the residual energy of the parents was equalized. (i.e.,) they would become $0.1 \mathrm{~J}$, $0.2 \mathrm{~J}$, and $0.2 \mathrm{~J}$, respectively.

The Energy required to transmit 1 bit of data from one sensor to another sensor can be calculated as follows

$$
\text { Prelay_S }=\left(\alpha 1+\alpha 2{ }^{*} \text { pow }(S, n)\right){ }^{*} \text { gamma }
$$

Where $\alpha 1$ is the total energy per bit consumer by the transmitter and receiver electronics. The total energy consumed is the energy consumed by the transmitter electronics and receiver electronics. Hence total energy per bit is $1 /$ total energy consumed. $\alpha 2$ is the energy dissipated in transmitter op-amp. $\mathrm{S}$ is the distance between the nodes. Gamma is the number of bits transmitted per second. The energy consumed at parent node $\mathrm{j}$ for transmitting $\mathrm{I}$ bit of data is calculated as follows.

$$
\Delta E i j=\alpha 1+\alpha 2 * \operatorname{pow}(K j R, n) * I i j
$$

Where KjR denotes the number of neighbors in the transmission range and $\mathrm{Iij}$ denotes that $\mathrm{I}$ bit of data is transferred from node $\mathrm{i}$ to node $\mathrm{j}$.

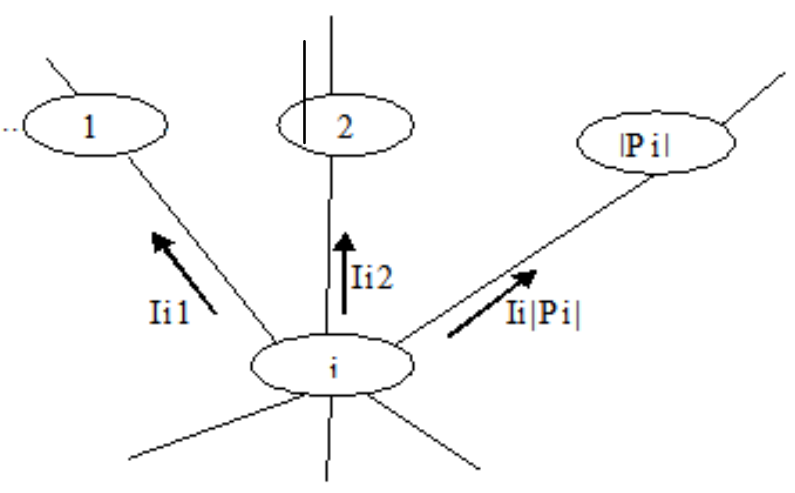

Fig-4: Multiple paths from node i to the sink 
After the data is transmitted, the residual energy of the parent node $\mathrm{j}$ is calculated as follows

$$
X j=E j-\Delta E i j
$$

Where $\mathrm{Ej}$ is the residual energy of node $\mathrm{j}$.

Node i calculates the total energy required to transmit I bit of data to its parents. It also calculates the average energy of all the capable parents for transmitting the data and sends the data only to the parents, whose energy is greater than the average. If a node $\mathrm{i}$ does not have a capable parent, it will be a isolated node.

Although the multipath transmission of data increases the network lifetime by balancing the energy of all the $\mathrm{CH}$ nodes, it increases the end-to-end delay and also decrease the throughput. The Backbone network with $\mathrm{CH}$ 's and member nodes with the sink at the top is shown in Fig-5.

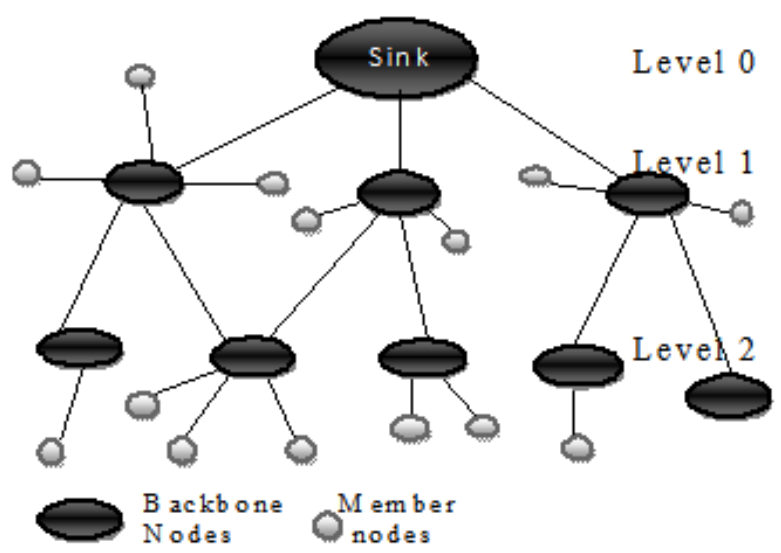

Fig-5: CH's at different levels with the sink.

The CH's at the lowest level collects the data from its members, perform data aggregation and send the data to its parents. But, the CH's at the higher level collects the data from its own members and also from the $\mathrm{CH}$ 's which is attached to it.

Hence the CH's at higher level have large amount of data than the CH's at lower level. By sending the data in multiple paths, end-to-end delay increases, which is due to the following reasons.

1. The wake up time is not set based on their distance from the sink

2. The wake up time is set as same for all the CH's in the network. But the $\mathrm{CH}$ which connects two isolated parts of network need to send more data than the normal node.

To overcome these,

1. The wake up time is set for the CH's based on the level it lies.
2. The wake up time is set based on the amount of data, which is determined by node's significance in connecting two different parts of network.

If the wake up time is same for all the CH's, then it increases end-to-end delay. Hence the delay aware wake up scheduling scheme is proposed in which, the wake-up time of CH's at higher level is set to be higher than the CH's at lower level. (i.e.,) The wake up time is inversely proportional to the distance from the sink.

\section{Algorithm}

Initialization: Set the wake up time to be normal for all $\mathrm{CH}$ nodes

For all the data which is to be sent do

Begin

Each $\mathrm{CH}$ determine its level based on the number of hops from the sink

if(level is 1)

The wake up time for $\mathrm{CH}$ is set to be 4 times the normal wake up time

else if (level is 2)

The wake up time for $\mathrm{CH}$ is set to be 3 times the normal wake up time

else if (level is 3)

The wake up time for $\mathrm{CH}$ is set to be 2 times the normal wake up time

else

The wake up time for $\mathrm{CH}$ is set to be normal

if $\mathrm{CH}$ connects two isolated parts of network)

Calculate the amount of data which is to be sent

Set the wake up time based on it

Each $\mathrm{CH}$ calculate its remaining energy

While (remaining energy > threshold value for head)

It will find the shortest energy density path to sink

Send the data in that path

If (energy of any $\mathrm{CH}$ is that path < threshold value for head)

That path is blocked and another alternate energy density path is found and send the data in the alternate energy density path End

Thus the end-to-end delay decreases and it also increase the network lifetime. Since the alternate shortest energy density path is chosen when energy of any $\mathrm{CH}$ in the selected shortest energy density path becomes less than the threshold, reliability is increased.

\subsubsection{Rerouting Algorithm}

In rerouting algorithm, the backbone is reconfigured only when the topology changes occur. It consists of 2 phases: Head replacement and isolated node reconnection. In head replacement phase, if the energy of the head exhausts or becomes lower than the threshold value, the exhausted head sends a help message to the neighbors. The waiting node has the higher priority to replace the exhausted head position. The waiting node is the node whose sensing range is entirely 
covered by its neighbor. If there exists more than one waiting node, then the node with the smallest id is chosen to become the head. If there are no waiting nodes in the exhausted head's neighbors, then the node with the high residual energy is chosen as a head.

In Isolated node reconnection phase, if the node is the $\mathrm{CH}$ and it does not have capable parents to transmit the data, it will become a isolated node. Hence it sends the reconnection message to its neighbors in order to connect to the backbone. If none of the backbone node responds, then increments the value of $\mathrm{k}$ and rebroadcasts the reconnection message. This process continues until it gets attached to the backbone node.

\section{PERFORMANCE EVALUATION}

The performance is evaluated using various parameters to show that the proposed algorithm (represented as green line) provides better efficiency than the existing algorithm(represented as red line). The lifetime of the network for different sense range when $1^{\text {st }}$ sensor node dies is shown in Fig-6, which describes that, the lifetime of proposed algorithm is higher than the existing algorithm.

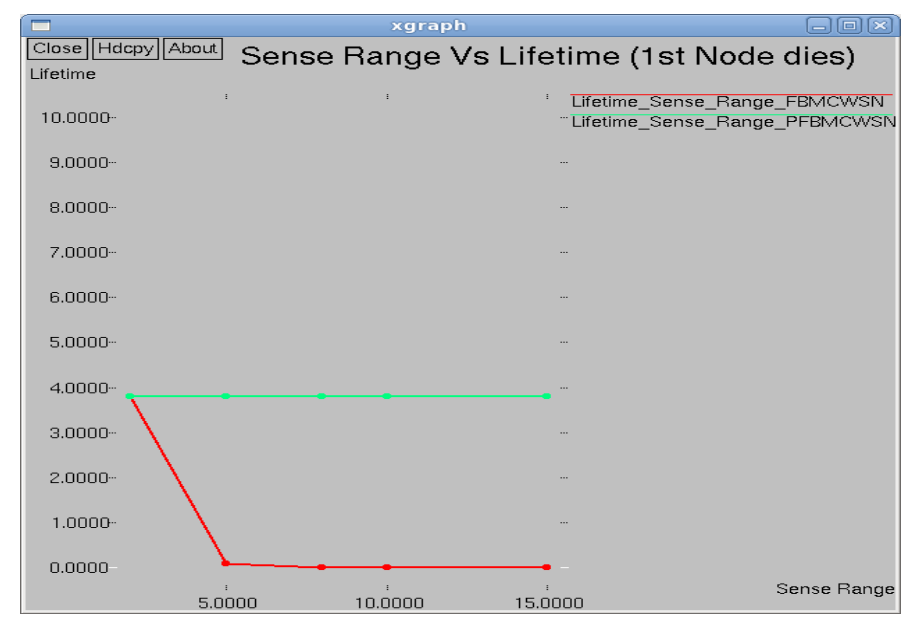

Fig-6: Network lifetime for different sense range when $1^{\text {st }}$ sensor node dies

The lifetime of the network for different sense range when $10 \%$ of sensor node dies is shown in Fig-7, which describes that, the lifetime of proposed algorithm is higher than the existing algorithm.

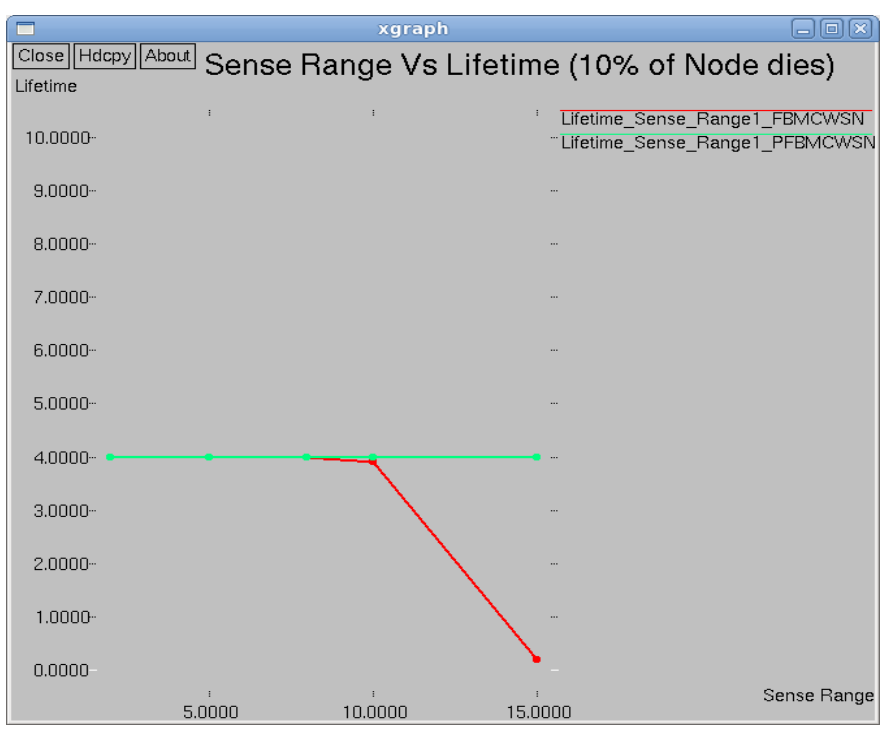

Fig-7: Network lifetime for different sense range when $1^{\text {st }}$ sensor node dies

The lifetime of the network for different cluster range when $1^{\text {st }}$ sensor node dies is shown in Fig-8, which describes that, the lifetime of proposed algorithm is higher than the existing algorithm.

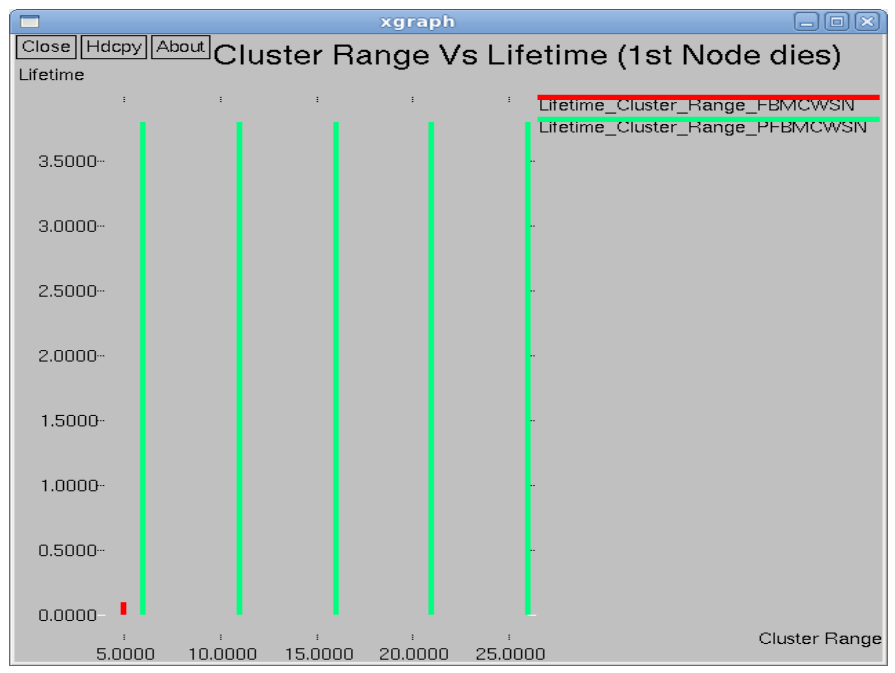

Fig-8: Network lifetime for different cluster range when $1^{\text {st }}$ sensor node dies

The lifetime of the network for different cluster range when $10 \%$ of sensor node dies is shown in Fig-9, which describes that, the lifetime of proposed algorithm is higher than the existing algorithm. 


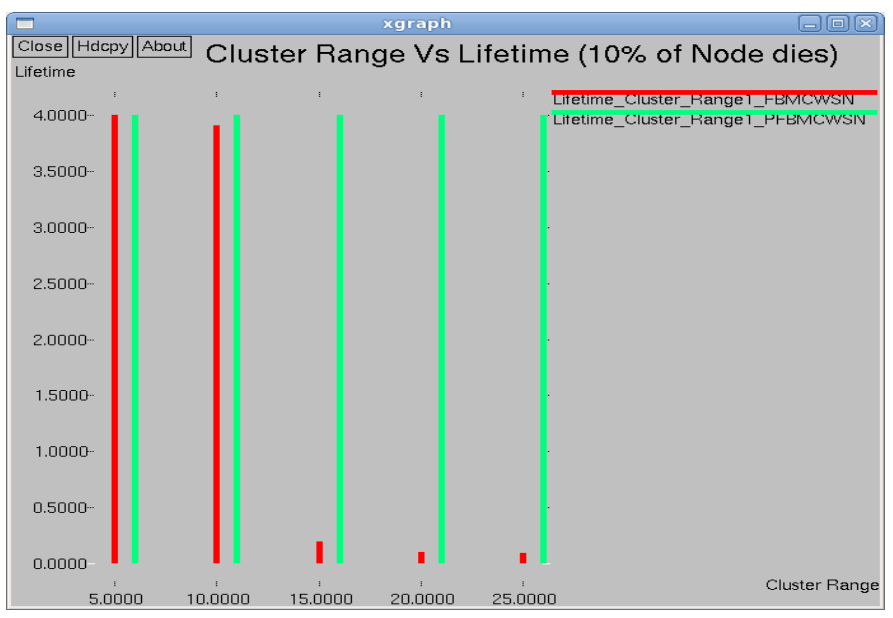

Fig-9: Network lifetime for different cluster range when $10 \%$ of sensor node dies

The Jitter for different time intervals is shown in Fig-10, which describes that, the Jitter in proposed algorithm is less than the existing algorithm.

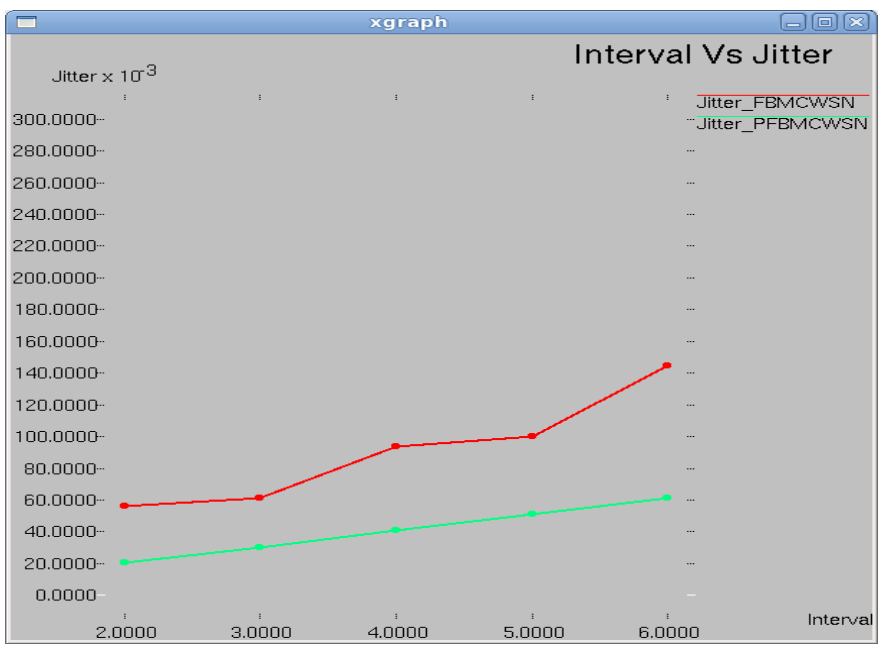

Fig-10: Jitter for different time intervals

\section{CONCLUSIONS}

In the existing algorithm for multi hop data transmission, the energy is balanced among the $\mathrm{CH}$ 's while transmitting the data to the sink which increases the network lifetime. But it increases the delay in transmitting the data. Hence the delay aware wake up scheduling scheme is proposed in which, the wake up time for CH's at the higher level is set to be higher than the CH's at the lower level which decrease the end to end delay and also increases the network lifetime. Simulation results illustrate that the proposed algorithm decreases the end to end delay and also increase the network lifetime.

\section{REFERENCES}

[1] I.F.Akyildiz, W.Su,Y.Sankarasubramaniam and E.Cayirci, Wireless Sensor Networks: A Survey, Computer Networks (Elsevier) Journal, vol.38, no.4, pp.393-422, March 2002.

[2] Wibhada Naruephiphat, Chalermpol Charnsripinyo, Clustering Techniques in Wireless Sensor Networks, in 2009 International Conference on New Trends in Information and Service Science,vol.3,no.5, April 2009.

[3] Ramesh Rajagopalan, Pramod K. Varshev: Dataaggregation techniques in sensor networks: A survey, IEEE Communications Surveys and Tutorials, vol.8,no.4,pp.48-63,January 2006.

[4] Kavi Khedo, Rubeena Doomun ,Sonum Aucharuz, READA: Redundancy Elimination for Accurate Data Aggregation in Wireless Sensor Networks, in scientific research Journal Wireless Sensor Networks,vol.2,no.4,pp.300-308, April 2010.

[5] Stanislava Soro, Wendi B. Heinzelman, CH election techniques for coverage preservation in wireless sensor networks, Ad Hoc Networks(Elsevier) Journal, vol.7, no.5,pp.955-972,July 2009.

[6] M. Perillo, W. Heinzelman, DAPR: a protocol for wireless sensor networks utilizing an application-based routing cost, in Proceedings of the IEEE Wireless Communications and Networking Conference (WCNC'04), November 2004.

[7] O. Younis, S. Fahmy, Distributed clustering in ad-hoc sensor networks: a hybrid, energy-efficient approach, IEEE Transactions on Mobile Computing,vol.3, no.4, January 2004.

[8] Tzong-Jye Liu,Hung-Wei Shen, Chia-Lin Lee, An Energy-Aware Backbone Construction Algorithm in Wireless Sensor Networks, in IEEE conference on Mobile Ad Hoc and Sensor Systems, April 2011.

[9] H. Raei, M. Sarram, F. Adibniya, F. Tashtarian,"Optimal distributed algorithm for minimum connected dominating sets in Wireless Sensor Networks", in IEEE conference on Mobile Ad Hoc and Sensor Systems, vol.2,no.3,pp. 695-700, June 2008.

[10] Jing (Selena) He, Shouling J, Pingzhi Fan, Yi Pan, Yingshu Li, Constructing a Load-Balanced Virtual Backbone in Wireless Sensor Networks, in International Conference on Computing, Networking and Communications, Wireless Ad Hoc and Sensor Networks Symposium,vol.4,no.6, pp.1-12,January 2012.

[11] Liang Xue, Xin-Ping Guan, Zhi-Xin Liu, Qing-Chao Zheng, A Power- and Coverage-aware Clustering Scheme for Wireless Sensor Networks, International Journal of Automation and Computing, vol.7,no.4,pp. 500-508, November 2010. 
[12] Tao Liu, Yunan Gu, Zhifeng Hu, Energy Equalizing Routing Algorithm for Wireless Sensor Networks, in Second International Symposium on Computational Intelligence and Design,vol.1, pp.546-549,January 2009.

[13] Bhaskar Bhuyan, Hiren Kumar Deva Sarma, Nityananda Sarma, Avijit Kar, Rajib Mall, Quality of Service (QoS) Provisions in Wireless Sensor Networks and Related Challenges, in scientific research Journal ,Wireless Sensor networks,vol.2,no.4,pp.861-868, November 2010.

[14] Vidya K S, Arun Anoop M, Lifetime Enhanced Cluster Based Routing in Wireless Sensor Networks, in International Journal of Engineering Science Invention, Vol.2,no.7, pp.69-72, June 2013.

[15] Alper Bereketli Ozgur B. Akan, Event-to-Sink Directed Clustering in Wireless Sensor Networks, in IEEE Communications Society,vol.1.no.5,pp.348-354,April 2009.

[16] Sumit Kumar, Siddhartha Chauhan,A Survey on Scheduling Algorithms for Wireless Sensor Networks, in International Journal of Computer Applications, vol.20, pp.0975-8887, April 2011.

[17] Yanwei Wu, Xiang-Yang Li, YunHao Liu, Wei Lou, Energy-Efficient Wake-Up Scheduling for Data Collection and Aggregation, in IEEE Transactions on parallel and distributed systems, vol. 21, no.2, February 2010.

[18] Babar Nazir, Halabi Hasbullah and Sajjad A Madani, Sleep/wake up scheduling scheme for minimizing endto-end delay in multi hop wireless sensor networks, in EURASIP Journal on Wireless Communications and Networking, vol.16, no.5, September 2011. 